\title{
Edaravone Protects against Pancreatic and Intestinal Injury after Acute Pancreatitis via Nuclear Factor- $\kappa$ B Signaling in Mice
}

\author{
Bingbin Wang and Wendong Lin* \\ Department of Anesthesiology, The First Affiliated Hospital of Wenzhou Medical University; Wenzhou 325003, China. \\ Received October 24, 2019; accepted December 12, 2019
}

Acute pancreatitis (AP) is one kind of acute surgical abdominal disease in the world. It causes intestinal damage with subsequent bacterial migration, endotoxemia and secondary pancreatic infections. In this investigation, we determined that edaravone (EDA) reduces pancreatic and intestinal injury after AP in mice. This was demonstrated by a reduction in histological score, apoptosis, interleukin (IL)-6, IL-1 $\beta$ and tumor necrosis factor (TNF)- $\alpha$, along with obstructing activation of Toll-like receptor 4 (TLR4) and nuclear factor- $\kappa \mathrm{B}(\mathrm{NF} \kappa \mathrm{B})$. Our study results suggested that EDA exerts its protective effects against pancreatic and intestinal injury after AP via regulation of the TLR4/NF $\mathrm{B}$ pathway. Our findings provide the basis for EDA to treat AP-induced pancreatic and intestinal injury, even might develop as a potential therapy for other inflammatory diseases.

Key words acute pancreatitis; edaravone; Toll-like receptor 4 (TLR4); nuclear factor- $\kappa \mathrm{B}(\mathrm{NF} \kappa \mathrm{B})$; inflammatory response

\section{INTRODUCTION}

Acute pancreatitis (AP) is an inflammatory response of various etiologies that leads to digestion, edema and necrosis of pancreatic tissue, and carries a high mortality. ${ }^{1)}$ The exact mechanism of AP is not fully understood. However, it is clear that the pathological processes of oxidative stress, ${ }^{2)}$ autophagy $^{3)}$ and especially of inflammation ${ }^{4)}$ are involved in AP disease progression. In the early stages of AP a large amount of inflammatory mediators are released, with excessive inflammatory cytokine exposure leading to damage of multiple organs throughout the body. Thus, inhibiting the inflammatory response may prevent progression of pancreatitis and improve its prognosis. Nuclear factor-kappaB $(\mathrm{NF} \kappa \mathrm{B})$ is an important transcription factor in the inflammatory response. ${ }^{5)}$ Prior work has shown that inhibiting NF $\kappa$ B activation can shorten the course of pancreatitis. ${ }^{6)}$ Thus, regulating $\mathrm{NF} \kappa \mathrm{B}$ expression may offer a treatment approach for pancreatitis. Toll-like receptor (TLR) is a typical receptor in the mechanism of innate immune responses. It can be activated to trigger signaling pathways involved in inflammation and mediate innate immune responses. TLR4 is also a key target for the regulation of the transcription factor $\mathrm{NF} \kappa \mathrm{B}$, which can further contribute to stimulate the expression of various cytokines. ${ }^{7)}$ Moreover, AP results in destruction of intestinal villus structure and increases the permeability of intestinal cells. $^{8)}$ Intestinal bacteria and harmful substances can penetrate the tissues around the intestinal tract, and even further activate immune cells to release inflammatory mediators and cytokines. ${ }^{9)}$ Therefore, early repair of intestinal injury is very beneficial to recovery from AP. Although treatment level of AP has been developed to some extent, the mortality rate of this disease remains very high. Currently, there is no reliably effective treatment for AP.

Edaravone (EDA, 3-methyl-1-phenyl-2-pyrazolin-5-one) scavenges free radicals and inhibits lipid peroxidation, as well as having antagonistic effects against apoptosis and cytokines. ${ }^{10-12)}$ At present, it is mainly used to treat cerebral infarction. Other work has also confirmed that EDA has a protective effect against dextran sulfate sodium (DSS)-induced colitis by promoting M2 macrophage polarization via the Janus kinase 2 (JAK2)-signal transducer and activator of transcription 3 (STAT3) signaling pathway. ${ }^{13)}$ It is not yet known whether EDA protects the intestine in a mouse model of AP. EDA has been shown to have a protective role in the mouse model of cerebral ischemia reperfusion injury ${ }^{14)}$ that is mediated by anti-inflammatory effects. However, few studies have evaluated the anti-inflammatory activity of EDA in AP.

The purpose of this study was to test the ability of EDA to attenuate AP-induced intestinal and pancreatic injury in mice.

\section{MATERIALS AND METHODS}

Animals and Model Wild-type C57BL/6 male mice were procured from the Animal Center of Wenzhou Medical University (Wenzhou, China). The animals were maintained at the optimum temperature along with $35-45 \%$ humidity and a $12: 12 \mathrm{~h}$ day-night cycle. Acclimatization was conducted for at least one week before any experimentation. The mice were fasted for $12 \mathrm{~h}$ prior to intervention. Intraperitoneal injections of $50 \mu \mathrm{g} / \mathrm{kg}$ cerulein (dissolved in sodium chloride $(0.9 \%)$ ) for 7 doses per hour was used to induce mice to develop as AP model. Cerulein is a cholecystokinin analogue, which can stimulate the secretion of pancreatic juice through blood circulation, and stimulate acinar cells to secrete trypsin. As a result, the digestive enzyme in the pancreas is activated in advance, causing the digestion of the pancreas itself. The advantage of cerulein-induced pancreatitis is that it does not require surgery and is suitable for studying the internal signal transduction pathways that may be involved in protease activation and cell death pathways, as well as to demonstrate the effectiveness of new treatments. Intraperitoneal injections of $6 \mathrm{mg} / \mathrm{kg}$ EDA was used to mice after AP, This dose of EDA administration was based on previous studies of protec- 
tive effect by EDA in AP model. ${ }^{15,16)}$ In addition, the dose of EDA used in this study $(6 \mathrm{mg} / \mathrm{kg})$ was higher than that in the clinical treatment (about $1 \mathrm{mg} / \mathrm{kg}$ ), and the reason for the difference in dose lies in that EDA is administered intravenously in clinical with a high bioavailability, and pancreatitis patients were generally treated by EDA combined with other drugs for several weeks. According to our and other previous studies, ${ }^{17,18)}$ inflammation levels were significantly upregulated following AP and peaked $24 \mathrm{~h}$ post-surgery in pancreatic tissue. Therefore, the mice were killed after $24 \mathrm{~h}$ from the first injection. Then conducted all the subsequent experiment on approval of the Animal Care and Use Committee of Wenzhou Medical University (Wenzhou, China).

Reagents and Chemicals Cerulein was purchased from Sigma Chemical (Sigma-Aldrich, St. Louis, MO, U.S.A.). Antibodies against $\mathrm{NF} \kappa \mathrm{B}$ p65, p-NF $\kappa \mathrm{B}$ IL-6, tumor necrosis factor (TNF)- $\alpha$ and TLR4 were purchased from Cell Signaling Technology (CST, Danvers, MA, U.S.A.). EDA was purchased from Lijun Pharmaceutical Co., Ltd, (Xi an, shan xi, China).

Western Blot Analysis The protein on the sodium dodecyl sulfate-polyacrylamide gel electrophoresis (SDS-PAGE) gel were firstly transferred to a polyvinylidene defluoride (PVDF) membrane (Bio-Rad Laboratories, Hercules, CA, U.S.A.) by electrophoresis. The membrane were then blocked with 5\% milk in Tris buffered saline tween (TBST) at room temperature for $2 \mathrm{~h}$ and incubated with the primary antibodies, anti-NF $\kappa \mathrm{B}(1: 1000)$ and anti-glyceraldehyde-3-phosphate dehydrogenase (GAPDH) $(1: 10000)$, at $4{ }^{\circ} \mathrm{C}$ overnight. Then the membranes were incubated with a secondary antibody at room temperature for $1 \mathrm{~h}$. The signals and densities of the immunoreactive bands were measured by the ChemiDicTM XRS+ Imaging System (BioRad Laboratories, Hercules, CA, U.S.A.).

Histological Examination The pancreas and intestinal tissue were collected and fixed in 4\% paraformaldehyde (PFA) for $24 \mathrm{~h}$ and then embedded in paraffin wax. Before the histopathological examination, samples were mounted onto poly L-lysine-coated slides and stained with hematoxylin. Images were obtained by a Nikon ECLIPSE 80i (Nikon, A1 PLUS, Tokyo, Japan). Intestinal histological damage was scored from 0 to 5 as described previously. ${ }^{19)}$ There were 5 grades were observed in intestinal tissue, normal (grade 0); subepithelial Gruenhagen's space (grade 1); the subepithelial space is enlarged (grade 2); epithelial lifting down and with a few denuded tips (grade 3); villi and lamina propria was denuded and angiotelectasis (grade 4); the lamina propria is destroyed (grade 5). The method of pancreatic histological scores were introduced previously. ${ }^{20)}$ Briefly, these criteria: acinar necrosis, edema, hemorrhage and inflammation and infiltration were assessed in pancreatic tissue. The score (0 (normal)-4 (severe)) was taken for each criterion to evaluate AP severity.

Real-Time Quantitative PCR (RT-qPCR) We used TRIzol (Invitrogen, Carlsbad, CA, U.S.A.) to extract total RNA from intestinal and pancreatic tissues, and the Prime Script RT-PCR kit (TaKaRa Bio, Dalian, China) for reverse transcription. RT-qPCR was amplified by CFX 96 TM realtime system (Bio-Rad Laboratories), the specific primer sequences (Invitrogen Shanghai, China) was as follows: mouse, TNF- $\alpha$ forward: TGA TCCGCGACGTGGAA, reverse: ACC GCC TGGAGT TCT GGA A; interleukin (IL)-6 forward: CCA AGA GGT GAG TGC TTC CC, reverse: CTGTTGTTC AGA CTC TCTCCC T; interleukin (IL)- $1 \beta$ forward: ACTCCT
TAG TCC TCGGCC A, reverse: CCA TCA GAGGCA AGG AGG AA; $\beta$-actin forward: CCGTGAAAA GATGACCCA GA, reverse: TAC GAC CAG AGG CAT ACA G.

Terminal Deoxynucleotidyl Transferase (TdT)-Mediated Deoxyuridine Triphosphate Nick-End Labeling (TUNEL) Staining In situ Cell Death Detection Kit (Roche, South San Francisco, CA, U.S.A.) was used to detect the apoptotic cells in the intestinal tissue. Positive cells were identified by the light microscope and analyzed by investigator blinded to the experimental groups.

Fluorescence Immunoassays After deparaffinization, rehydration and mounting on coverslips, these tissue section then were blocked for $30 \mathrm{~min}$ at $37^{\circ} \mathrm{C}$ with $5 \%$ bovine serum albumin (BSA), then incubated with anti-NFKB $(1: 300)$, anti-TLR4(1:300), anti-IL-6(1:200) and anti-TNF- $\alpha(1: 200)$ specific primary detection antibodies for overnight at $4{ }^{\circ} \mathrm{C}$. The slides were then incubated with the secondary antibody $(1: 1000)$ for $2 \mathrm{~h}$ at room temperature. After washed with PBS, slides were re-stained with 4'-6-diamidino-2-phenylindole (DAPI) for $7 \mathrm{~min}$. Fluorescence was observed with a Nikon confocal laser microscope (Nikon).

Statistical Analysis All data are presented as mean \pm standard error of the mean (S.E.M.). GraphPad Pro 5.0 software (San Diego, CA, U.S.A.) was used for statistical analyses. One-way ANOVA was used to test the significance of multiple groups, and $p$-values $<0.05$ were defined as significance. All experiments were performed for at least three times.

\section{RESULTS}

EDA Decreased Pancreatic and Intestinal Damage after AP in Mice To evaluate the therapeutic effect of EDA in AP-associated pancreatic and intestinal injury, we assessed the pathological changes after AP. In HE staining assay, edema and leukocyte infiltration occurred in the non-treated AP group but the sham group appeared to have a normal structure, whereas the EDA group had more normal pathological features than the AP group in pancreatic (Fig. 1A) and intestinal tissues (Fig. 1B). The pathological scores were higher in the AP group than in the sham group, while EDA treatment resulted in the scores decreasing in pancreatic (Fig. 1C) and intestinal tissues (Fig. 1D).The immunofluorescence analysis of cleaved-caspase3 showed a significant lower expression of cleaved-caspase 3 proteins in the EDA treatment group compared with the AP group in intestinal tissues (Fig. 1F). Correspondingly, EDA treatment reduces TUNEL-positive apoptotic cells in AP group in pancreatic tissues (Fig. 1E).

EDA Suppressed AP-Induced Inflammatory Response To measure whether the protective effect of EDA was due to its ability to suppress the inflammatory response induced by AP, we determined the expression of IL- 6 and TNF- $\alpha$ in the pancreas and intestinal tissue. RT-qPCR analysis demonstrated that the expression of TNF- $\alpha$, IL- 6 and IL- $1 \beta$ at the mRNA level was higher in the AP group than in the sham group, but the cytokines decreased in the EDA groups after EDA treatment (Figs. 2A-G). Immunostaining of IL-6 and TNF- $\alpha$ illustrated that the expression of IL- 6 and TNF- $\alpha$ were higher in the AP group compared with the sham group, while EDA treatment resulted in reduction of the expression of IL- 6 and TNF- $\alpha$ (Fig. 2D). 


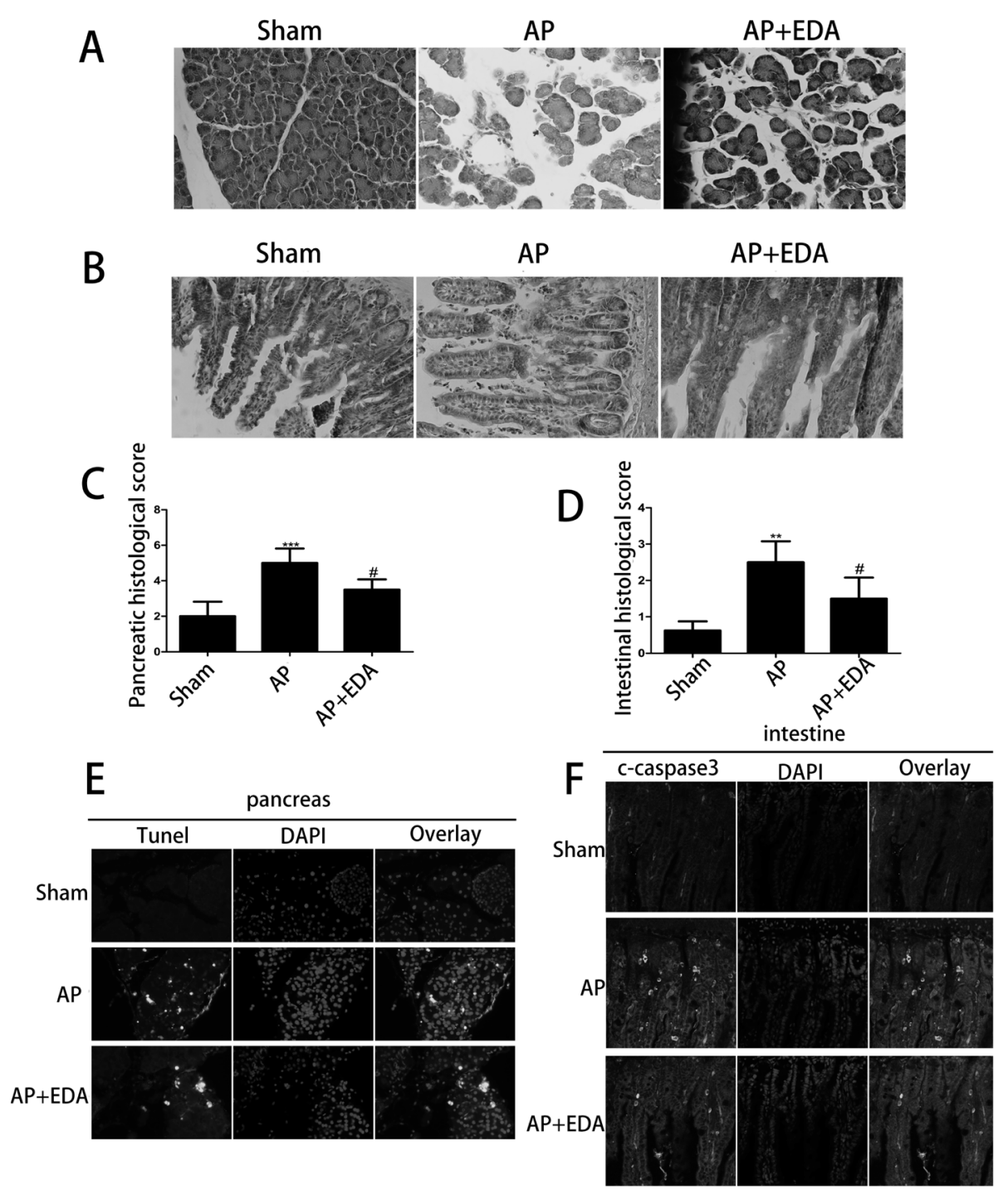

Fig. 1. EDA Treatment Protects against Cerulein-Induced AP in Mice

(A, B) Pancreatic and intestinal morphological changes in the different groups at $24 \mathrm{~h}$ after AP. (C) Pancreatic histological scores. (D) Intestinal histological scores. (E) The result of TUNEL assay in the pancreatic tissue. (F) Immunofluorescence staining of C-caspase3. Results are expressed as mean \pm S.E.M. $n=5$ per group. *** $p<0.001$ and $* * p<0.01$ versus sham group. ${ }^{\#} p<0.05$ versus AP group.

EDA Inhibited the Expression of NFKB Protein after AP in Mice $\mathrm{NF} \kappa \mathrm{B}$ activity is essential for an inflammatory response and our previous research showed that EDA treatment significantly decreased IL- 6 and TNF- $\alpha$ expression. Thus, we further examined the effects of EDA on $\mathrm{NF} \kappa \mathrm{B}$ activation after $\mathrm{AP}$. $\mathrm{NF} \kappa \mathrm{B}$ and $\mathrm{p}-\mathrm{NF} \kappa \mathrm{B}$ expression were increased in pancreatic and intestinal tissue in the AP group compared with the untreated sham group, and was significantly inhibited by EDA (Figs. 3A, B, H, I). Besides, lower TLR4 expression was observed in EDA treatment group compared to untreated controls (Figs. 3C-F). This result was also demonstrated by immunofluorescence analysis of TLR4 (Fig. 3G).

\section{DISCUSSION}

At present, patients suffering from acute pancreas mainly receive non-surgical treatment, and the specific drugs for acute pancreatitis remain scarce. In the pancreatitis model induced by cerulein, trypsinogen is activated to produce trypsin to induce acinar cell injury, where a variety of pro- inflammatory cytokines are induced via various TLR receptors, thus aggravating the pancreatic injury. ${ }^{21)}$ A study has shown that the mice lacking the TLR4 gene have less pancreatic damage when treated with cerulein-induced AP than the wild-type mice. ${ }^{22}$ Furthermore, the activation of TLR4 expressed in pancreatic acinar cells can promote the secretion of IL- $1 \beta$, which further alters intestinal permeability and causes other manifestations of pancreatitis. ${ }^{23)}$ Previous study has also shown that activation of inflammatory responses and bacterial translocation after intestinal injury are major risk factors for $\mathrm{AP}^{24,25)}$ In this context, we considered that intervening in the pathogenesis of AP would be an effective way to treat it. EDA has a significant beneficial effect in liver injury $^{26)}$ and in cerebral ischemia. ${ }^{14)}$ EDA has also been shown to alleviate Oxidative stress and apoptosis of the intestinal epithelium after burns was shown to be alleviated by EDA via the mitogen-activated protein kinase (MAPK) pathway. ${ }^{27)} \mathrm{We}$ thus investigated the possible effects of EDA in pancreatic and intestinal tissue with mouse model of AP. We found that EDA protects against pancreatic and intestinal injury via inhibition 

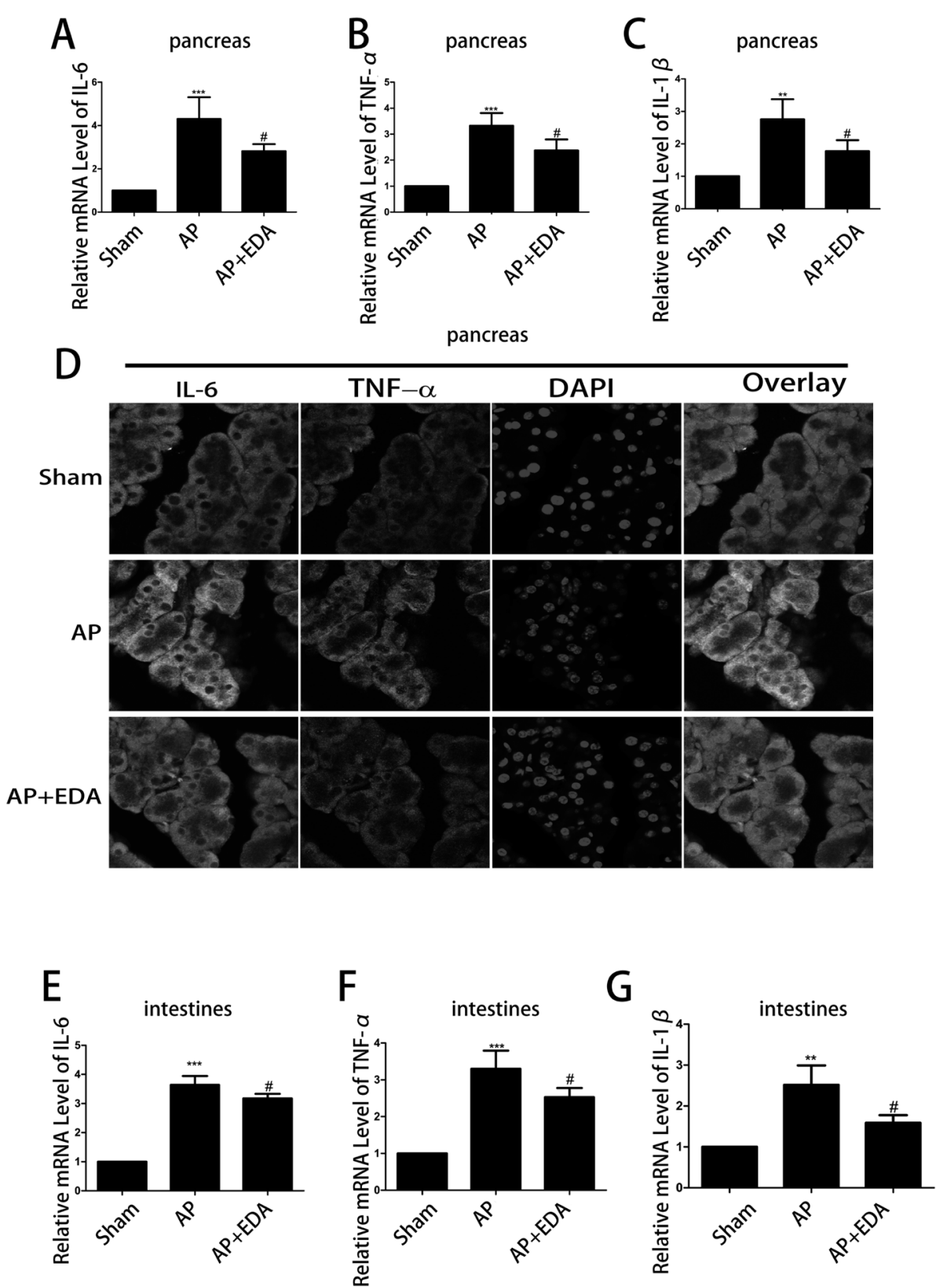

Fig. 2. EDA Treatment Reduces Inflammatory Response after AP

(A-C) IL-6, TNF- $\alpha$ and IL-1 $\beta$ mRNA levels in pancreatic tissue were analysed by RT-qPCR in the different groups. (D) Immunofluorescence staining of IL-6 and TNF- $\alpha$ in pancreatic tissue. (E-G) TNF- $\alpha$, IL-6 and IL-1 $\beta$ mRNA levels in intestinal tissue were analysed by RT-qPCR in the different groups. Results are expressed as mean \pm S.E.M. $n=5$ per group. ${ }^{* * *} p<0.001,{ }^{* *} p<0.01$ versus sham group. ${ }^{\sharp} p<0.05$ versus AP group.

of the inflammatory response.

$\mathrm{NF} \kappa \mathrm{B}$ is closely involved in the inflammatory response. In our previous research, EDA has been suggested on the anti-inflammatory effects in AP and has been demonstrated to relieve lipopolysaccharide (LPS)-induced acute lung injury in mice by suppressing NF $\kappa$ B activation. ${ }^{28)}$ Therefore, we inferred that EDA's protective effect in AP-induced injury may be realized through the $\mathrm{NF} \kappa \mathrm{B}$ signaling pathway. TLR4, as a key receptor that aggravates AP injury, can up-regulate the expressions of inflammatory mediators via receiving ligand signals. ${ }^{29,30)}$ On the one hand, when AP occurs, the abnormal activation of intracellular trypsin can lead to local pancreatic injury and cell necrosis. Moreover, protein molecules in the necrotic cells can enter extracellular fluid and be recognized as endogenous ligands by TLR4 to initiate immune response and sterile inflammatory response. ${ }^{29)}$ On the other hand, the intestinal mucosal barrier injury caused by AP can cause intestinal conditions pathogenic bacteria to shift into the blood circulation, and the resulting exogenous ligands are recognized by TLR4, start the NF $\kappa$ B pathway, and up-regulate the expressions of inflammatory mediators such as cytokines and chemokines. ${ }^{30)}$ Some studies show that the protective effect of edaravone against acute lung injury, ${ }^{31)}$ skeletal muscles, ${ }^{32)}$ acute kidney injury ${ }^{33)}$ and acute stroke ${ }^{34)}$ associated with the $\mathrm{NF} \kappa \mathrm{B}$ activation and inflammatory cytokine production. In addition, other studies point to EDA inhibition of mRNA levels of TLR4 and NF $\kappa$ B expression in cerebral ischemia-reperfusion in mice. In AP, we determined that EDA treatment suppressed activation of $\mathrm{NF}_{\mathcal{B}} \mathrm{B}$ protein in the pancreas and intestines. Activation of TLR4/NFkB pathway also cause the 
A
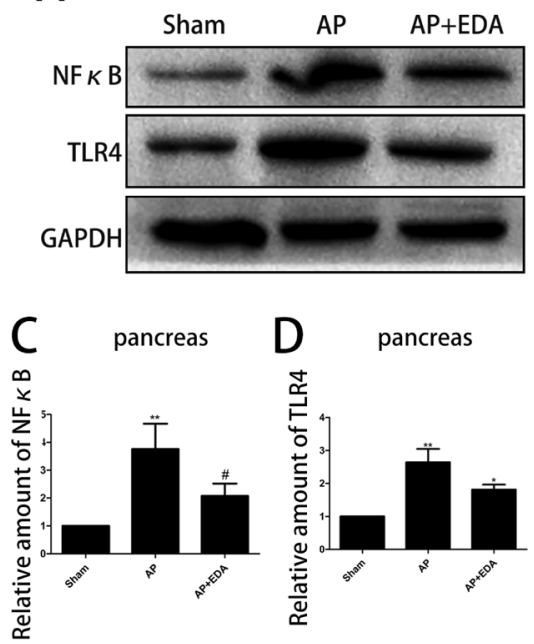

G

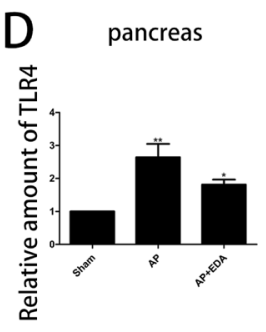

TLR4
B

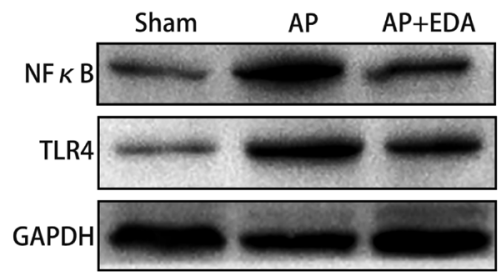

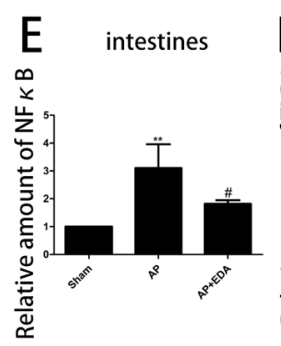

Overlay

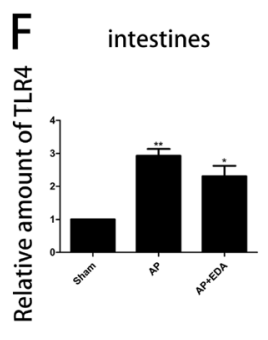

DAPI
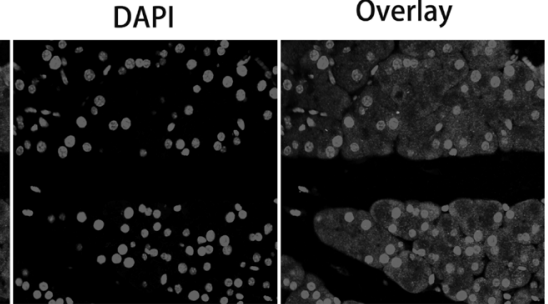

Sham
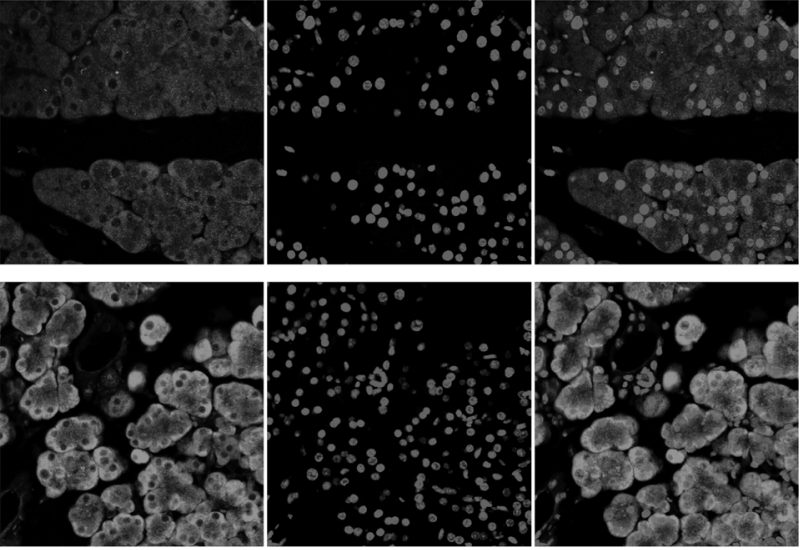

AP
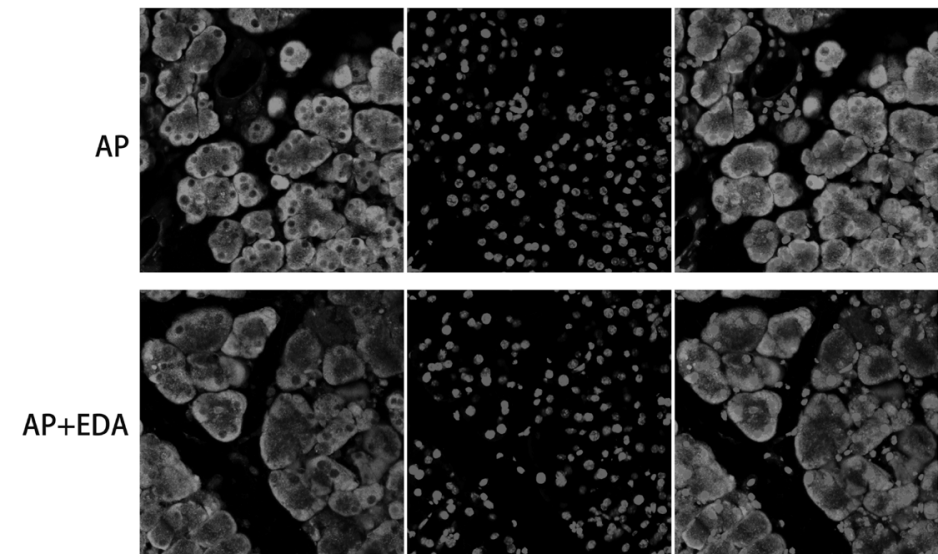
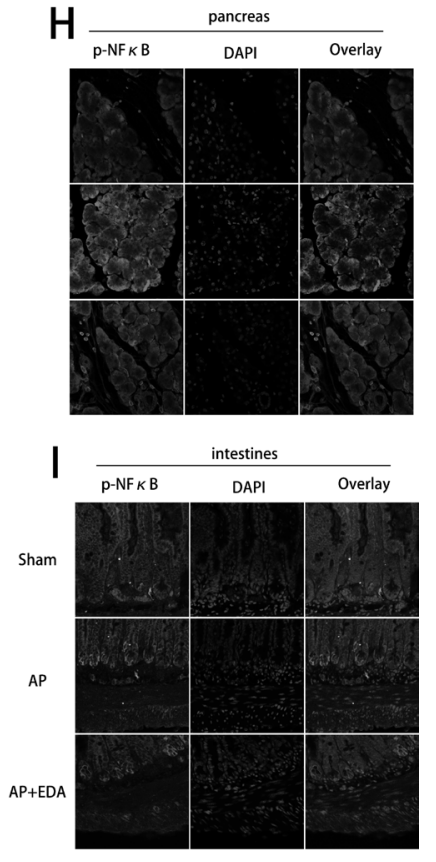

Fig. 3. Effect of EDA Treatment on $\mathrm{NF} \kappa \mathrm{B}$ in Pancreatic and Intestinal Tissue after AP in Mice

(A, B) Protein expression of TLR4 and NF $\kappa$ B in pancreatic and intestinal tissue after AP in different groups. GAPDH was as the loading control for band density normalization. (C-F) Statistical graph of TLR4 and NF $\kappa$ B TLR4 protein in the different groups. (G) Immunofluorescence staining of TLR4 in intestinal tissue. (H, I). Immunofluorescence staining of $\mathrm{p}-\mathrm{NF} \kappa \mathrm{B}$. Results are expressed as mean \pm S.E.M. $n=5$ per group. $* * p<0.01$ versus sham group. ${ }^{\#} p<0.05$ versus AP group.

release of a large number of inflammatory factors, which can lead to excessive inflammatory response of the body. Therefore, the study about the mechanism on regulation the Pancreatitis may be further induced by TLR4/NF- $\kappa \mathrm{B}$ pathway in LPS-stimulated pancreatic cells. ${ }^{35)}$ We found that in the mouse model of AP, inflammatory response could be restrained by EDA treatment, to some extent, regulated by theTLR4/ NF $\kappa \mathrm{B}$ pathway.

In a healthy intestinal tract, bacteria from the intestinal lumen cannot enter the blood circulation due to the intestinal mucosal barrier and other structures. ${ }^{36)}$ During the initiation and development of AP, intestinal function is impaired and permeability is increased. Harmful bacteria enter pancreatic tissue through the damaged intestinal mucosa, aggravating pancreatic infection, and eventually leading to multiple organ failure. ${ }^{37)}$ Therefore, protecting intestinal function will be beneficial to recovery from AP. A previous report has demonstrat- ed that EDA mitigates ischemic mucosal damage after acute superior mesenteric artery thromboembolism in a rabbit autologous fibrin clot model. ${ }^{38)}$ Therefore, we give the investigation on the effects of EDA on intestinal injury in a mouse model of AP. Our results proved that EDA can suppress edema and leukocyte infiltration in the intestine after AP.

In conclusion, we give the evidence that EDA significantly reduced pancreatic and intestinal damage in a mouse model of AP by attenuating the inflammatory response. Furthermore, we demonstrated that EDA repairs pancreatic and intestinal injury via regulation of the $\mathrm{TLR} 4 / \mathrm{NF} \kappa \mathrm{B}$ signal pathway. These findings were thus on the basis of therapeutic strategies with EDA that could assist with the recovery from AP in clinical practice.

Acknowledgments Funding from the program of Wenzhou Municipal Science and Technology Bureau (No. 2016Y0394). 
Conflict of Interest The authors declare no conflict of interest.

\section{REFERENCES}

1) Chatila AT, Bilal M, Guturu P. Evaluation and management of acute pancreatitis. World J. Clin. Cases, 7, 1006-1020 (2019).

2) $\mathrm{Yu} \mathrm{JH}, \mathrm{Kim} \mathrm{HY}$. Oxidative stress and inflammatory signaling in cerulein pancreatitis. World J. Gastroenterol., 20, 17324-17329 (2014).

3) Wan J, Yang X, Ren Y, Li X, Zhu Y, Haddock AN, Ji B, Xia L, Lu N. Inhibition of miR-155 reduces impaired autophagy and improves prognosis in an experimental pancreatitis mouse model. Cell Death Dis., 10, 303 (2019).

4) Rafaz H, Ahsan F, Malik FG, Wajahat ZM. Sterile inflammatory response in acute pancreatitis. Pancreas, 41, 353-357 (2012).

5) Hoyles L, Snelling T, Umlai U-K, Nicholson JK, Carding SR, Glen RC, McArthur S. Microbiome-host systems interactions: protective effects of propionate upon the blood-brain barrier. Microbiome, $\mathbf{6}$, 55 (2018)

6) Zhou Y, Zhao L, Mei F, Hong Y, Xia H, Zuo T, Ding Y, Wang W. Macrophage migration inhibitory factor antagonist $(S, R) 3($ 4hydroxyphenyl)4,5dihydro5isoxazole acetic acid methyl ester attenuates inflammation and lung injury in rats with acute pancreatitis in pregnancy. Mol Med. Rep., 17, 6576-6584 (2018).

7) Bhatelia K, Singh K, Singh R. TLRs: linking inflammation and breast cancer. Cell. Signal., 26, 2350-2357 (2014).

8) Sun X, Shao Y, Jin Y, Huai J, Zhou Q, Huang Z, Wu J. Melatonin reduces bacterial translocation by preventing damage to the intestinal mucosa in an experimental severe acute pancreatitis rat model. Exp. Ther. Med., 6, 1343-1349 (2013).

9) Miao YF, Kang HX, Li J, Zhang YM, Ren HY, Huan LZ, Yuan $\mathrm{CL}, \mathrm{Su} \mathrm{H}$, Wan MH, Tang WF. Effect of Sheng-jiang powder on multiple-organ inflammatory injury in acute pancreatitis in rats fed a high-fat diet. World J. Gastroenterol., 25, 683-695 (2019).

10) Li Z, Ma QQ, Yan Y, Xu FD, Zhang XY, Zhou WQ, Feng ZC. Edaravone attenuates hippocampal damage in an infant mouse model of pneumococcal meningitis by reducing HMGB1 and iNOS expression via the Nrf2/HO-1 pathway. Acta Pharmacol. Sin., 37, 1298-1306 (2016).

11) Qiang X, Li Y, Yang X, Luo L, Xu R, Zheng Y, Cao Z, Tan Z, Deng Y. DL-3- $n$-Butylphthalide-edaravone hybrids as novel dual inhibitors of amyloid-beta aggregation and monoamine oxidases with high antioxidant potency for Alzheimer's therapy. Bioorg. Med. Chem. Lett., 27, 718-722 (2017).

12) Tian A, Ma H, Zhang R, Cui Y, Wan C. Edaravone improves spatial memory and modulates endoplasmic reticulum stress-mediated apoptosis after abdominal surgery in mice. Exp. Ther. Med., 14, 355-360 (2017).

13) Zhang X, Xu F, Liu L, Feng L, Wu X, Shen Y, Sun Y, Wu X, Xu Q. (+)Borneol improves the efficacy of edaravone against DSS-induced colitis by promoting M2 macrophages polarization via JAK2STAT3 signaling pathway. Int. Immunopharmacol., 53, 1-10 (2017).

14) Chiazza F, Chegaev K, Rogazzo M, Cutrin JC, Benetti E, Lazzarato L, Fruttero R, Collino M. A nitric oxide-donor furoxan moiety improves the efficacy of edaravone against early renal dysfunction and injury evoked by ischemia/reperfusion. Oxid. Med. Cell Longev., 2015, 804659 (2015).

15) Fukudome D, Matsuda M, Kawasaki T, Ago Y, Matsuda T. The radical scavenger edaravone counteracts diabetes in multiple lowdose streptozotocin-treated mice. Eur. J. Pharmacol., 583, 164-169 (2008).

16) Zhou CH, Lin L, Zhu XY, Wen-Tang, Hu DM, Dong Y, Li LY, Wang SF. Protective effects of edaravone on experimental chronic pancreatitis induced by dibutyltin dichloride in rats. Pancreatology, 13, 125-132 (2013).

17) Fan HN, Chen W, Fan LN, Wu JT, Zhu JS, Zhang J. Macrophages- derived p38alpha promotes the experimental severe acute pancreatitis by regulating inflammation and autophagy. Int. Immunopharmacol., 77, 105940 (2019).

18) Vrolyk V, Schneberger D, Le K, Wobeser BK, Singh B. Mouse model to study pulmonary intravascular macrophage recruitment and lung inflammation in acute necrotizing pancreatitis. Cell Tissue Res., 378, 97-111 (2019).

19) Deng W, Abliz A, Xu S, Sun R, Guo W, Shi Q, Yu J, Wang W. Severity of pancreatitisassociated intestinal mucosal barrier injury is reduced following treatment with the NADPH oxidase inhibitor apocynin. Mol. Med. Rep., 14, 3525-3534 (2016).

20) Schmidt J, Rattner DW, Lewandrowski K, Compton CC, Mandavilli U, Knoefel WT, Warshaw AL. A better model of acute pancreatitis for evaluating therapy. Annals of Surgery, 215, 44-56 (1992).

21) Cen Y, Liu C, Li X, Yan Z, Kuang M, Su Y, Pan X, Qin R, Liu X, Zheng J, Zhou H. Artesunate ameliorates severe acute pancreatitis (SAP) in rats by inhibiting expression of pro-inflammatory cytokines and Toll-like receptor 4. Int. Immunopharmacol., 38, 252-260 (2016).

22) Sharif R, Dawra R, Wasiluk K, Phillips P, Dudeja V, Kurt-Jones E, Finberg R, Saluja A. Impact of toll-like receptor 4 on the severity of acute pancreatitis and pancreatitis-associated lung injury in mice. Gut, 58, 813-819 (2009).

23) Pan LF, Yu L, Wang LM, He JT, Sun JL, Wang XB, Wang H, Bai $\mathrm{ZH}$, Feng H, Pei HH. Augmenter of liver regeneration (ALR) regulates acute pancreatitis via inhibiting HMGB1/TLR4/NF $\kappa$ B signaling pathway. Am. J. Transl. Res., 10, 402-410 (2018), J.

24) Hallac A, Puri N, Applebury D, Myers K, Dhumal P, Thatte A, Srikureja W. The value of quick sepsis-related organ failure assessment scores in patients with acute pancreatitis who present to emergency departments: a three-year cohort study. Gastroenterology Res., 12, 67-71 (2019).

25) Pagliari D, Brizi MG, Saviano A, Mancarella FA, Dal Lago AA, Serricchio ML, Newton EE, Attili F, Manfredi R, Gasbarrini A. Clinical assessment and management of severe acute pancreatitis: a multi-disciplinary approach in the XXI century. Eur. Rev. Med. Pharmacol. Sci., 23, 771-787 (2019).

26) Fujiwara N, Som AT, Pham LD, Lee BJ, Mandeville ET, Lo EH, Arai K. A free radical scavenger edaravone suppresses systemic inflammatory responses in a rat transient focal ischemia model. Neurosci. Lett., 633, 7-13 (2016).

27) $\overline{\mathrm{Wu} J}$, Zhou M, Yu X, Ma J, Wu Y. Edaravone alleviates oxidative stress and apoptosis of intestinal epithelium after burns via MAPK pathway. Minerva Med., 110, 587-590 (2019).

28) Zhang Z, Luo Z, Bi A, Yang W, An W, Dong X, Chen R, Yang S, Tang H, Han X, Luo L. Compound edaravone alleviates lipopolysaccharide (LPS)-induced acute lung injury in mice. Eur. J. Pharmacol., 811, 1-11 (2017).

29) Zhang XP, Zhu CM, Wu DJ, Jiang X. Possible Role of Toll-Like Receptor 4 in Acute Pancreatitis. Pancreas, 39, 819-824 (2010).

30) Vaz J, Akbarshahi H, Andersson R. Controversial role of toll-like receptors in acute pancreatitis. World J. Gastroenterol., 19, 616-630 (2013).

31) Zhang Z, Luo Z, Bi A, Yang W, An W, Dong X, Chen R, Yang S, Tang H, Han X, Luo L. Compound edaravone alleviates lipopolysaccharide (LPS)-induced acute lung injury in mice. Eur. J. Pharmacol., 811, 1-11 (2017).

32) Yokoyama $\mathrm{H}$, Tsujii $\mathrm{M}$, Iino $\mathrm{T}$, Nakamura $\mathrm{T}$, Sudo A. Inhibitory effect of edaravone on systemic inflammation and local damage in skeletal muscles following long-term ischemia to murine hind limb. J. Orthop. Surg., 27, 2309499019874470 (2019).

33) Liu L, Song Y, Zhao M, Yi Z, Zeng Q. Protective effects of edaravone, a free radical scavenger, on lipopolysaccharide-induced acute kidney injury in a rat model of sepsis. Int. Urol. Nephrol., 47, 1745-1752 (2015).

34) Fujiwara N, Som AT, Pham LD, Lee BJ, Mandeville ET, Lo EH, 
Arai K. A free radical scavenger edaravone suppresses systemic inflammatory responses in a rat transient focal ischemia model. Neurosci. Lett., 633, 7-13 (2016).

35) Pan LF, Yu L, Wang LM, He JT, Sun JL, Wang XB, Wang H, Bai $\mathrm{ZH}$, Feng H, Pei HH. Augmenter of liver regeneration (ALR) regulates acute pancreatitis via inhibiting HMGB1/TLR4/NF- $\kappa \mathrm{B}$ signaling pathway. Am. J. Transl. Res., 10, 402-410 (2018).

36) Zhang M, Zhu HM, He F, Li BY, Li XC. Association between acute pancreatitis and small intestinal bacterial overgrowth assessed by hydrogen breath test. World J. Gastroenterol., 23, 8591-8596 (2017).
37) Zhang XM, Zhang ZY, Zhang CH, Wu J, Wang YX, Zhang GX, Intestinal microbial community differs between acute pancreatitis patients and healthy volunteers. Biomed. Environ. Sci., 31, 81-86 (2018).

38) Sonoda A, Nitta N, Seko A, Ohta S, Takemura S, Sugimoto T, Uzu T, Yokomaku Y, Takahashi M, Kashiwagi A, Murata K. Does the concomitant intra-arterial injection of asialoerythropoietin and edaravone mitigate ischaemic mucosal damage after acute superior mesenteric artery thromboembolism in a rabbit autologous fibrin clot model? Br. J. Radiol., 83, 129-132 (2010). 